\title{
Open Reduction of Proximal Interphalangeal Fracture-Dislocation through a Midlateral Incision Using Absorbable Suture Materials
}

\author{
Jae Jun Lee, Hyoung Joon Park, Hyun Gon Choi, Dong Hyeok Shin, Ki Il Uhm \\ Department of Plastic and Reconstructive Surgery, Konkuk University School of Medicine, Seoul, Korea
}

\begin{abstract}
Background Fracture-dislocation of the proximal interphalangeal (PIP) joint is a relatively common injury. Various treatments for fracture-dislocation of the PIP joint have been reported. In the present study, we performed open reduction through a midlateral incision using absorbable sutures to reduce the small bone fragments and performed volar plate repair.

Methods We treated nine patients with fracture-dislocation of the PIP joint with small fractured bone fragments too small for pinning or screw fixation. Patients with volar plate injury were treated with open reduction and volar plate repair at the periosteum of the middle phalangeal bone base by the modified Kessler method using absorbable sutures. All patients were placed in a dorsal aluminum extension block splint, which maintained the PIP joint in approximately 30 degrees of flexion to avoid excessive tension on the sutured volar plate.

Results At a mean final follow-up of postoperative 9 months, all patients were evaluated radiographically and had adequate alignment of the PIP joint and reduction of the displaced bone fragments. Range of motion was improved and there were no complications.

Conclusions This technique is an excellent alternative to the current method of treating patients with fracture-dislocations that include small fragments that are too small for pinning or screw fixation. It is a less invasive surgical method and enables stable reduction and early exercise without noticeable complications.
\end{abstract}

Keywords Finger joint / Intra-articular fractures / Volar plate / Sutures
Correspondence: Hyoung Joon Park Department of Plastic and Reconstructive Surgery, Konkuk University School of Medicine, 120 Neungdong-ro, Gwangjin-gu, Seoul 143-729, Korea

Tel: +82-2-2030-8191

Fax: $+82-2-2030-5249$

E-mail: paolian@naver.com

This article was presented at the 70th Congress of the Korean Society of Plastic and Reconstructive Surgeons on November 9, 2012 in Seoul, Korea.

No potential conflict of interest relevant to this article was reported.

Received: 20 Feb 2013 • Revised: 8 Apr 2013 • Accepted: 29 Apr 2013

pISSN: 2234-6163 • elSSN: 2234-6171 • http://dx.doi.org/10.5999/aps.2013.40.4.397 • Arch Plast Surg 2013;40:397-402

\section{INTRODUCTION}

Dorsal fracture-dislocation of the proximal interphalangeal (PIP) joint is a relatively common injury that most frequently occurs as a result of axial force applied to an extended finger [1]. Hyperextension of the PIP joint of the fingers results in a spectrum of injuries, ranging from incomplete disruptions of the volar plate and collateral ligaments to unstable fractures and dislocations. Ruptures of the volar capsular mechanism usually occur at the base of the middle phalanx, often with small fragments of the middle phalangeal bone $[2,3]$. The primary concerns associated with this fracture-dislocation are joint stability and maintenance of a concentric reduction. If the fracture-dislocation is not treated properly, it may lead to instability, degenerative arthritis, stiffness, and persistent pain [4]. Various therapeutic methods including splinting [5], pinning [6,7], open reduction and internal fixation [8-10], volar plate arthroplasty [11], external fixation [12], and hemi-hamate autograft [13] have been reported

Copyright $\odot 2013$ The Korean Society of Plastic and Reconstructive Surgeons

This is an Open Access article distributed under the terms of the Creative Commons Attribution Non-Commercial License (http://creativecommons.org/

licenses/by-nc/3.0/) which permits unrestricted non-commercial use, distribution, and reproduction in any medium, provided the original work is properly cited.

www.e-aps.org 
in the past. In certain cases, however, closed reduction cannot be performed due to the small size of the fragments, or there is hesitation in recommending invasive treatment because of the extent of the injury. In the present study, we performed open reduction through a midlateral incision using polydioxanone (PDS, Ethicon, San Angelo, TX, USA) absorbable sutures and performed volar plate repair. This technique allows for a more accurate reduction of the small bone fragments under direct visualization through a midlateral incision with simultaneous repair of the volar plate.

\section{METHODS}

\section{Patients}

Between April 2011 and October 2012, 9 patients (8 men and 1 women; mean age, 31 years) with fracture-dislocation of the PIP joint visited our department. For these patients, accurate reduction could not be performed with pinning or screw fixation because of the small size of the bone fragments. Patients were categorized before the surgery through physical examinations and radiographic assessments. Patients were selected as the study subjects if they maintained stability after reduction despite radiography images showing displaced bone fragments that induced pain upon passive movement and flexion limitation upon active movement.

For 5 patients, only the volar plate was ruptured and for the other 4 patients, the unilateral collateral ligament as well as the volar plate were concomitantly ruptured. The dominant hand was involved in 2 patients and the non-dominant hand in 7 patients. Three injuries resulted from falls, and 6 patients were injured by a ball while playing sports. The fracture-dislocation site was the PIP joint of the ring finger in 2 cases, the PIP joint of the little finger in 6, and the PIP joint of the index finger in 1 (Table 1). All patients were operated on within 1 week of injury. The injured fingers were protected by splinting until the day of operation. Postoperative radiographs were taken immediately and serially.

\section{Surgical methods}

The procedure was performed under general anesthesia, with tourniquet control. A midlateral skin incision of approximately $2 \mathrm{~cm}$ was made over the PIP joint. Incisions were made on the ulnar side for the index finger and on the radial side for the ring and little fingers.

The dissection was performed with caution to avoid any injuries to the neurovascular bundle. The ruptured volar plate was then exposed by careful traction on the flexor tendon. The volar plate was completely ruptured in the vicinity of the distal attachment site of the middle phalangeal bone base, which included the proximal avulsed fragments from the middle phalangeal bone. The ruptured volar plate with the small bone fragment was repaired using 5-0 PDS sutures. The suture was passed through the periosteum of the middle phalangeal bone base using the modified Kessler method. Reduction of the small bone fragment was achieved by suture of the volar plate (Figs. 1, 2). In some cases, multiple small bone fragments were observed. In these cases, the very small bone flecks were removed and only those fragments attached to the volar plate were reduced; additional pull-out sutures were not required in all cases.

After accurate reduction, stability was confirmed by gently extending the PIP joint; we also checked the surrounding soft tissue for blood vessel, nerve, and ligament injury. The skin was then sutured using 5-0 polyamide (Dafilon, B.Braun Melsungen AG, Melsungen, Germany) without intradermal suturing. In patients with concomitant damage of the volar plate and collateral ligament after repair of the volar plate by the procedure described, the ruptured collateral ligament was repaired with PDS 5-0 suture, using a figure-of-eight method.

\section{Postoperative care}

A dorsal aluminum extension block splint was applied to maintain the PIP joint at 30 degrees of flexion. After the operation,

\section{Table 1. Patient characteristics}

\begin{tabular}{|c|c|c|c|c|}
\hline Case No. & Sex/Age (yr) & Vector & Location & Operative finding \\
\hline 1 & $M / 34$ & Sports injury & Right 4th PIP joint, fracture dislocation & Volar plate avulsion \\
\hline 2 & $M / 26$ & Sports injury & Left 5th PIP joint, fracture dislocation & Volar plate avulsion \\
\hline 3 & $M / 16$ & Sports injury & Left 5th PIP joint, fracture dislocation & Volar plate avulsion \\
\hline 4 & $M / 18$ & Sports injury & Left 5th PIP joint, fracture dislocation & Volar plate avulsion \\
\hline 5 & $\mathrm{M} / 48$ & Sports injury & Right 5th PIP joint, fracture dislocation & Volar plate avulsion \\
\hline 6 & $M / 24$ & Slip down & Left 5th PIP joint, fracture dislocation & Volar plate avulsion, collateral ligament rupture \\
\hline 7 & $\mathrm{~F} / 43$ & Sports injury & Left 5th PIP joint, fracture dislocation & Volar plate avulsion, collateral ligament rupture \\
\hline 8 & $M / 17$ & Slip down & Left 4th PIP joint, fracture dislocation & Volar plate avulsion, collateral ligament rupture \\
\hline 9 & $M / 58$ & Slip down & Left 2nd PIP joint, fracture dislocation & Volar plate avulsion, collateral ligament rupture \\
\hline
\end{tabular}


complications such as hypoesthesia, hematoma, and infection were not detected. Splinting was performed for 3 weeks and the splint was extended by 10 degrees each week. After the splint was removed, active range of motion (ROM) was implemented. During outpatient follow-up, radiographs were performed to verify maintenance of reduction, and the functional outcome and ROM were determined.

\section{Evaluation methods}

Means and standard deviations were assessed for functional outcomes at preoperative and postoperative follow-up points in time. Results were evaluated using the records of the evaluation of ROM. Statistical analysis using SPSS ver. 17 (SPSS Inc., Chicago, IL, USA) was performed for the postoperative complications, and the results were compared with those of other studies. The statistical test used was the Wilcoxon signed rank test. Sta-

\section{Fig. 1. Schematic drawings of surgical technique}

(A) A midlateral skin incision (red line) is made over the proximal interphalangeal joint. (B) The ruptured volar plate is exposed. (C)

Primary repair of the ruptured volar plate with small bone fragments is done.
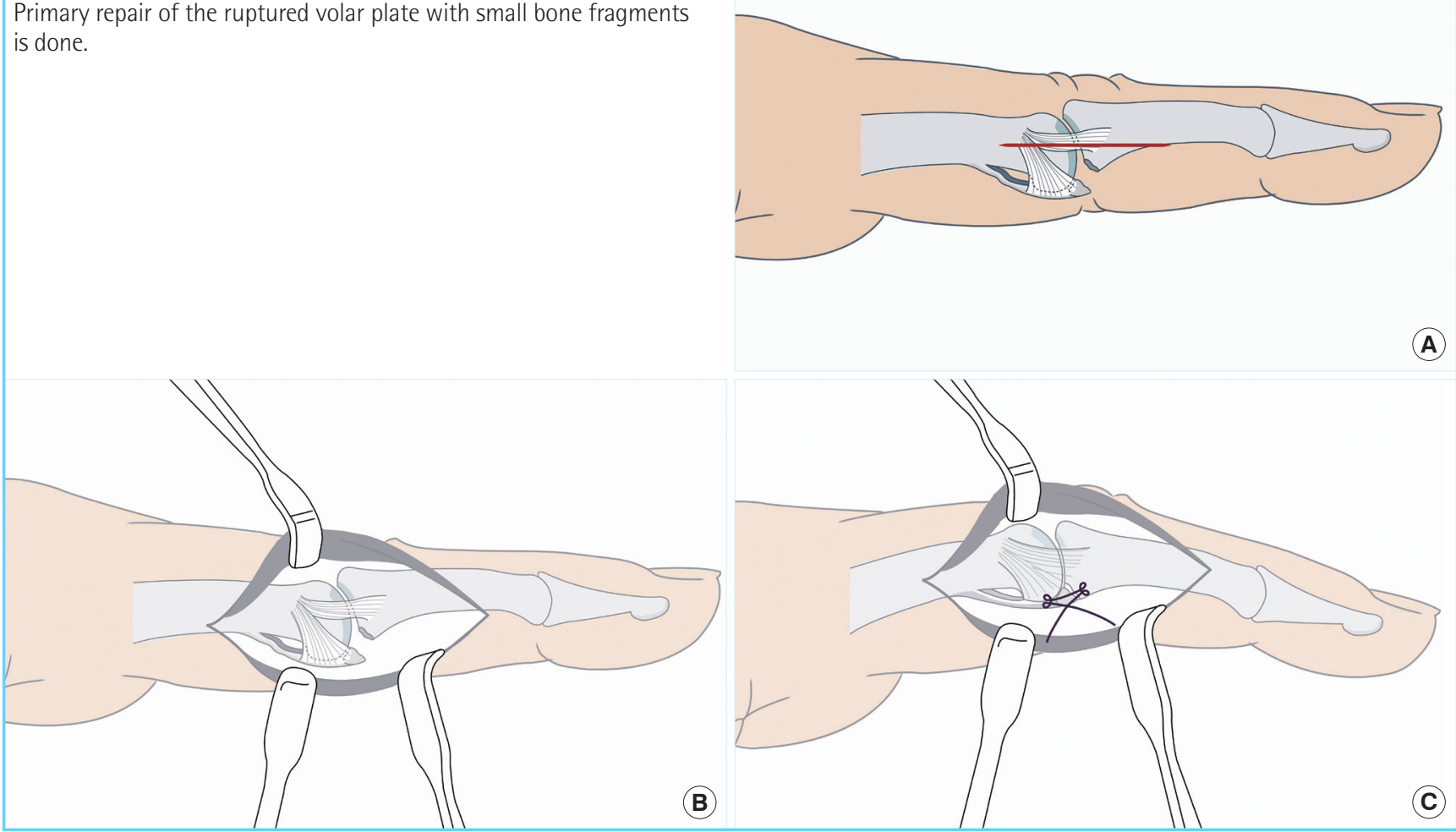

\section{Fig. 2. Intraoperative photographs of surgical technique}

(A) The ruptured volar plate is exposed by careful traction to the flexor tendon. (B) Primary repair of the ruptured volar plate with small bone fragments is done by suturing with 5-0 polydioxanone.
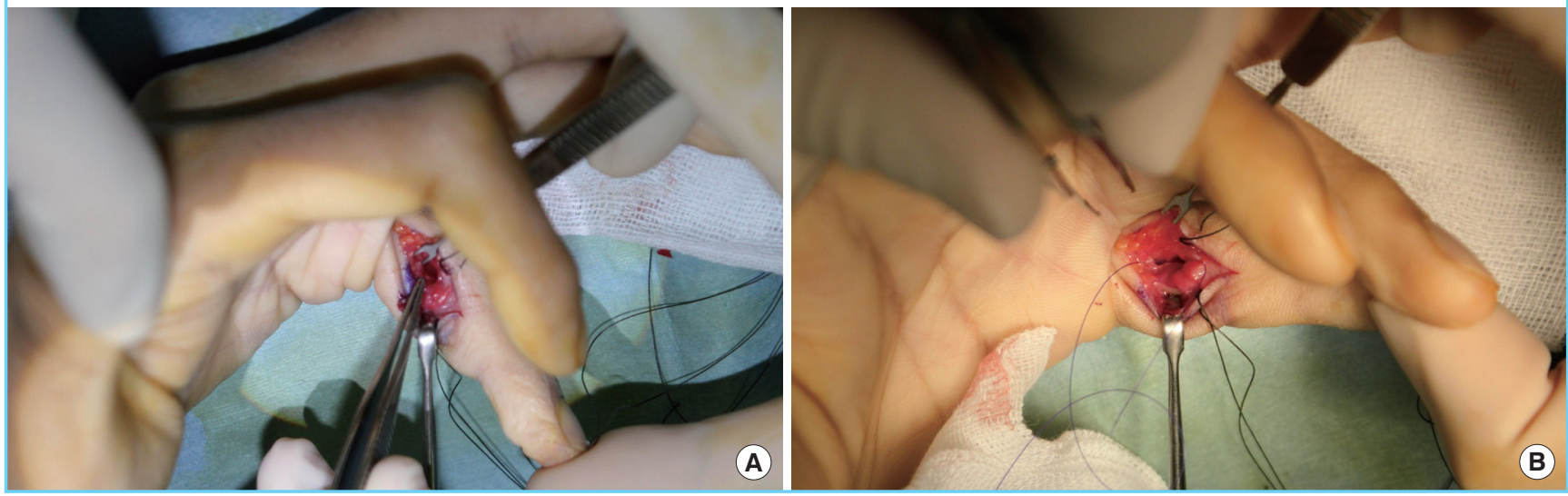


\section{Table 2. Summary of results}

\begin{tabular}{|c|c|c|c|c|c|c|}
\hline \multirow{2}{*}{ Case No. } & \multirow{2}{*}{ Sex/Age (yr) } & \multirow{2}{*}{ Injured finger } & \multicolumn{3}{|c|}{ Range of motion $\left({ }^{\circ}\right)$} & \multirow{2}{*}{$\begin{array}{l}\text { Follow-up } \\
\quad(\mathrm{mo})\end{array}$} \\
\hline & & & Preoperative & Postoperative & Improved & \\
\hline 1 & $M / 34$ & Ring & 52 & 91 & 39 & 13 \\
\hline 2 & $M / 26$ & Little & 81 & 95 & 14 & 12 \\
\hline 3 & $M / 16$ & Little & 65 & 94 & 29 & 10 \\
\hline 4 & $M / 18$ & Little & 20 & 90 & 70 & 11 \\
\hline 5 & $\mathrm{M} / 48$ & Little & 50 & 96 & 46 & 11 \\
\hline 6 & $M / 24$ & Little & 35 & 93 & 58 & 6 \\
\hline 7 & $\mathrm{~F} / 43$ & Little & 30 & 90 & 60 & 6 \\
\hline 8 & $\mathrm{M} / 17$ & Ring & 85 & 100 & 15 & 4 \\
\hline 9 & $M / 58$ & Index & 60 & 90 & 30 & 4 \\
\hline
\end{tabular}

Table 3. Mean ROM of the PIP joint

\begin{tabular}{|c|c|c|c|}
\hline & \multicolumn{2}{|c|}{ Mean ROM ( $\left.{ }^{\circ}\right)$} & \multirow{2}{*}{ P-value } \\
\hline & Preoperative & Postoperative & \\
\hline PIP joint & $53.1 \pm 22.2$ & $93.2 \pm 3.4$ & 0.008 \\
\hline
\end{tabular}

tistical significance was assumed for $\mathrm{P}<0.05$.

\section{RESULTS}

The follow-up period was 9 months on average (range, 4 to 13 months). Follow-up radiographs at 3 weeks after the operation confirmed that the bone fragments were reduced and the alignment of the PIP joint was well maintained. As the alignment of the PIP joint was maintained, the splint was removed under the judgment that fixation of the volar plate and collateral ligament were also well maintained. During outpatient follow-up, redislocation, subluxation, and nonunion were not observed (Fig. 3). Postoperative edema improved without any specific problems within 3 months. Most patients complained of postoperative inconvenience or pain that was alleviated by administering pain medications. Joint stiffness that transiently occurred after splint removal disappeared within 2 weeks. No patients complained of displacement of the PIP joint or sustained pain. In addition, complications such as intrinsic tightness, flexion contracture, and extension lag were not detected. The mean ROM of the PIP joint was enhanced from a preoperative value of 47.5 degrees to a postoperative value of 92.7 degrees (Table 2), and the difference was statistically significant (Table 3).

In addition, postoperative scarring was minimal; the scar size was approximately $2 \mathrm{~cm}$ because of the midlateral incision. The patients were satisfied with the result, as the scar was hidden between the fingers. No movement restrictions from scarring were found.

\section{Fig. 3. Preoperative and follow-up radiographs}

(A) Preoperative radiograph. (B) Follow-up image at 4 months shows bone union and joint congruity.
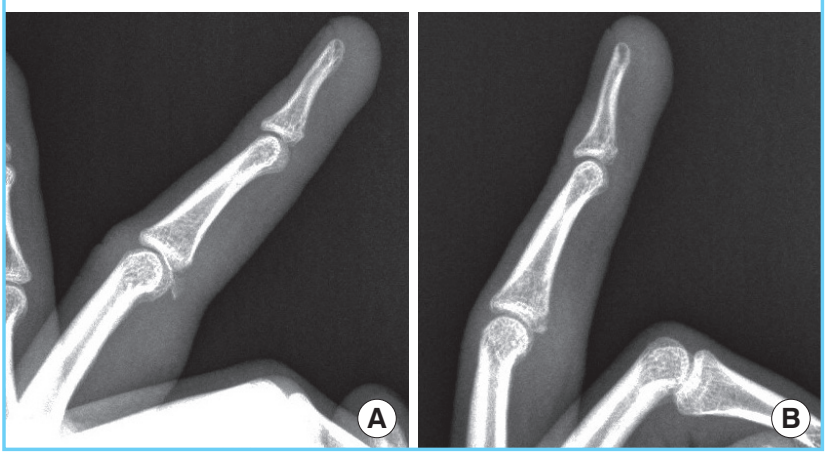

In the present case series, there was no postoperative subluxation or dislocation, and radiographs confirmed concentric reduction of the PIP joint in all cases.

\section{DISCUSSION}

Stability is determined by the size of the middle phalangeal volar base fragment, the degree of impaction of the articular surface, and, ultimately, by the ability to maintain a concentric reduction of the PIP joint during healing of the fracture [14].

Many patients require formal hand therapy to regain full motion and function. Patients and physicians aim at a perfect outcome. In the absence of specific treatment guidelines for stable volar plate lesions, some physicians tend to follow the general recommendations of textbooks on fracture-dislocations, particularly when a chip avulsion is visible in the radiograph, and therefore choose prolonged immobilization with a splint to treat these injuries. Blocking extension can maintain joint congruity but does not anatomically reduce fractures [15]. The volar fracture fragment may be trapped within the flexor sheath and inhibit motion [16]. The adverse effects of lengthy immobilization and the exclusion from activities that require hand motion are 
disregarded. The propensity of the PIP joints to develop flexion contractures is well known. These contractures are not only difficult to treat but may persist and predispose the patient to further injuries [17].

Newington et al. [6] more recently described their technique for closed reduction and percutaneous pinning in a series of 10 patients. The authors performed a closed reduction and then stabilized the PIP joint in flexion with a transarticular K-wire. They reported a mean ROM of the PIP joint of 85 degrees with a mean extension deficit of 8 degrees.

Another percutaneous technique for the treatment of these injuries employs the concept of dorsal block pinning. Dorsal block pinning [7] involves placing a smooth K-wire into the head of the proximal phalanx in lieu of a splint to prevent dorsal subluxation of the middle phalanx. Viegas [7] treated 3 patients, and at an average follow-up of 7 weeks the mean flexion at the PIP joint was 83 degrees with an extension deficit of 12 degrees. This procedure also has a limitation, in that an extension deficit remains as the mean ROM of the PIP joint is partially recovered.

Complications from these techniques, such as infection including osteomyelitis, pin loosening, pin migration, loss of reduction, nonunion, impaled flexor tendon, pseudarthrosis, and neurovascular injury can be encountered [18].

There are several alternative treatments of dorsal fracture-dislocations of the PIP joint. These include open reduction and internal fixation with K-wires [8], cerclage wires [9], interfragmentary screws [10], volar plate arthroplasty [11], dynamic external fixation [12], and hemi-hamate autograft reconstruction [13]. Each of these techniques has certain merits and drawbacks, but no surgical procedure has been shown to be superior $[1,19,20]$.

In this study, the authors repaired volar plates and collateral ligaments using PDS, an absorbable suture material. Using sutures made of nonabsorbable materials may cause stitch granuloma, fibrosis, triggering, and other complications because the body responds to the foreign suture material [21]. Kang et al. [22] repaired the flexor tendons using PDS on 55 fingers of 41 patients. No complications such as the aforementioned complications of nonabsorbable suture materials were detected. The rate of complications such as repair rupture or adhesion was similar for PDS and nonabsorbable suture materials. Furthermore, less restriction of ROM and more satisfactory functional recovery were obtained. With this background, we chose to use PDS for the operations.

There are some limitations to our study. First, it was a small case series. Second, given the small sample size, we did not attempt to compare the results of this technique with other techniques. A prospective, randomized trial comparing various techniques would be useful.
Open reduction with volar plate repair through a midlateral incision using absorbable sutures is a relatively simple surgical method for patients with PIP joint fracture-dislocation in which there are small bone fragments that are difficult to repair by pinning or screw fixation because of their small size. In addition, it is thought that a learning curve on the part of the surgeon is necessary to perform this surgery because of limited visibility and the narrow surgical area; however, this surgery possesses many advantages because it has a less invasive technique and results in fewer scars than that by volar incision. This method is an effective surgical procedure because stabilized reduction could be achieved, early exercise is allowed, and its complications are limited.

\section{REFERENCES}

1. Ng CY, Oliver CW. Fractures of the proximal interphalangeal joints of the fingers.J Bone Joint Surg Br 2009;91:705-12.

2. Bowers WH, Wolf JW Jr, Nehil JL, et al. The proximal interphalangeal joint volar plate. I. An anatomical and biomechanical study. J Hand Surg Am 1980;5:79-88.

3. Bowers $\mathrm{WH}$. The proximal interphalangeal joint volar plate. II: a clinical study of hyperextension injury.J Hand Surg Am 1981;6:77-81.

4. O’Rourke SK, Gaur S, Barton NJ. Long-term outcome of articular fractures of the phalanges: an eleven year follow up. J Hand Surg Br 1989; 14:183-93.

5. McElfresh EC, Dobyns JH, O’Brien ET. Management of fracture-dislocation of the proximal interphalangeal joints by extension-block splinting. J Bone Joint Surg Am 1972; 54:1705-11.

6. Newington DP, Davis TR, Barton NJ. The treatment of dorsal fracture-dislocation of the proximal interphalangeal joint by closed reduction and Kirschner wire fixation: a 16-year follow up. J Hand Surg Br 2001;26:537-40.

7. Viegas SF. Extension block pinning for proximal interphalangeal joint fracture dislocations: preliminary report of a new technique. J Hand Surg Am 1992;17:896-901.

8. Takami H, Takahashi S, Ando M. Large volar plate avulsion fracture of the base of the middle phalanx with rotational displacement: a report of three cases. J Hand Surg Am 1997; 22:592-5.

9. Weiss AP. Cerclage fixation for fracture dislocation of the proximal interphalangeal joint. Clin Orthop Relat Res 1996; (327):21-8.

10. Ford DJ, el-Hadidi S, Lunn PG, et al. Fractures of the phalanges: results of internal fixation using $1.5 \mathrm{~mm}$ and $2 \mathrm{~mm}$ A. O. screws. J Hand Surg Br 1987;12:28-33. 
11. Eaton RG, Malerich MM. Volar plate arthroplasty of the proximal interphalangeal joint: a review of ten years' experience. J Hand Surg Am 1980;5:260-8.

12. Ruland RT, Hogan CJ, Cannon DL, et al. Use of dynamic distraction external fixation for unstable fracture-dislocations of the proximal interphalangeal joint. J Hand Surg Am 2008; 33:19-25.

13. Williams RM, Kiefhaber TR, Sommerkamp TG, et al. Treatment of unstable dorsal proximal interphalangeal fracture/ dislocations using a hemi-hamate autograft. J Hand Surg Am 2003;28:856-65.

14. Kiefhaber TR, Stern PJ. Fracture dislocations of the proximal interphalangeal joint. J Hand Surg Am 1998;23:368-80.

15. Calfee RP, Sommerkamp TG. Fracture-dislocation about the finger joints. J Hand Surg Am 2009;34:1140-7.

16. Failla JM. Extrusion of fracture fragment into flexor sheath following proximal interphalangeal joint fracture-dislocation: a case report. J Hand Surg Am 1996;21:253-5.

17. Incavo SJ, Mogan JV, Hilfrank BC. Extension splinting of palmar plate avulsion injuries of the proximal interphalangeal joint. J Hand Surg Am 1989; 14:659-61.

18. Botte MJ, Davis JL, Rose BA, et al. Complications of smooth pin fixation of fractures and dislocations in the hand and wrist. Clin Orthop Relat Res 1992;276:194-201.

19. Deitch MA, Kiefhaber TR, Comisar BR, et al. Dorsal fracture dislocations of the proximal interphalangeal joint: surgical complications and long-term results. J Hand Surg Am 1999;24:914-23.

20. Aladin A, Davis TR. Dorsal fracture-dislocation of the proximal interphalangeal joint: a comparative study of percutaneous Kirschner wire fixation versus open reduction and internal fixation. J Hand Surg Br 2005;30:120-8.

21. Pabari A, Iyer S, Branford OA, et al. Palmar granuloma following flexor tendon repair using Ticron: a case for absorbable suture material? J Plast Reconstr Aesthet Surg 2011;64: 409-11.

22. Kang HJ, Lee DC, Kim JS, et al. Flexor tenorrhaphy using absorbable suture materials. Arch Plast Surg 2012;39: 397-403. 